\title{
Turbulence Mitigation Methods and their Evaluation
}

\author{
Adam W.M. van Eekeren", Judith Dijk, Klamer Schutte \\ TNO, PO Box 96864, 2509 JG The Hague, The Netherlands
}

\begin{abstract}
In general, long range detection, recognition and identification in visual and infrared imagery are hampered by turbulence caused by atmospheric conditions. The amount of turbulence is often indicated by the refractive-index structure parameter $C_{n}{ }^{2}$. The value of this parameter and its variation is determined by the turbulence effects over the optical path. Especially along horizontal optical paths near the surface (land-to-land scenario) large values and fluctuations of $C_{n}^{2}$ occur, resulting in an extremely blurred and shaky image sequence. Another important parameter is the isoplanatic angle, $\theta_{0}$, which is the angle where the turbulence is approximately constant. Over long horizontal paths the values of $\theta_{0}$ are typically very small; much smaller than the field-of-view of the camera.

Typical image artefacts that are caused by turbulence are blur, tilt and scintillation. These artefacts occur often locally in an image. Therefore turbulence corrections are required in each image patch of the size of the isoplanatic angle. Much research has been devoted to the field of turbulence mitigation. One of the main advantages of turbulence mitigation is that it enables visual recognition over larger distances by reducing the blur and motion in imagery. In many (military) scenarios this is of crucial importance. In this paper we give a brief overview of two software approaches to mitigate the visual artifacts caused by turbulence. These approaches are very diverse in complexity. It is shown that a more complex turbulence mitigation approach is needed to improve the imagery containing medium turbulence. The basic turbulence mitigation method is only capable of mitigating low turbulence.
\end{abstract}

Keywords: Turbulence mitigation, deconvolution, image restoration

\section{INTRODUCTION}

In general, long range detection, recognition and identification in visual and infrared (video) imagery are hampered by turbulence caused by atmospheric conditions. Especially over long ground-to-ground ranges atmospheric effects are dominant. An extensive statistical analysis of the turbulence effects scintillation, blur and beam wander over horizontal paths in a littoral environment are presented in de Jong et al. ${ }^{1}$. Relevance of turbulence effects at infrared wavelength is provided in a statistical study by Schwering and $\mathrm{Kunz}^{2}$. These atmospheric effects result in blurry and shaky images, which can be reduced by turbulence mitigation methods. Turbulence mitigation is a challenging problem because 1) the amount of turbulence is spatially varying and can be assumed constant only in a small local neighborhood and 2) the amount of turbulence varies significantly over time.

Typical approaches to mitigate the effects of turbulence are described $\mathrm{in}^{3,4}$. Basically there exist three approaches: 1) a hardware approach, 2) a software approach and 3) a combination of both approaches. The main advantages of a software approach are that it is flexible, cheap and effective, especially given the processing power available nowadays. In this paper we will focus on two software approaches that are developed within TNO. The first method consists of global motion compensation and Dynamic Super-Resolution (DSR) and is designed for processing imagery with low turbulence (typical $C_{n}{ }^{2} \sim 5.10^{-16} \mathrm{~m}^{-2 / 3}$ ). The second method consists of local motion compensation, blur estimation and unsharp masking and is designed for processing imagery with medium turbulence (typical $C_{n}^{2} \sim 5.10^{-14} \mathrm{~m}^{-2 / 3}$ ).

In an earlier paper ${ }^{5}$ we compared two different turbulence mitigation methods (TNO and Fraunhofer IOSB) on a few image sequences containing the same amount of turbulence. In this paper we will describe two TNO turbulence mitigation methods and we will qualitatively evaluate their performance on three different image sequences containing varying amounts of turbulence. It is shown that a basic turbulence mitigation approach is only capable of mitigating low

*adam.vaneekeren@tno.nl; www.tno.nl

Electro-Optical and Infrared Systems: Technology and Applications XI, edited by

David A. Huckridge, Reinhard Ebert, Proc. of SPIE Vol. 9249, 924900 · (c) 2014

SPIE · CCC code: $0277-786 X / 14 / \$ 18 \cdot$ doi: $10.1117 / 12.2067327$

Proc. of SPIE Vol. 9249 924900-1 
turbulence, while a more complex turbulence mitigation approach is needed to improve the imagery containing medium turbulence.

The setup of the paper is as follows. Section 2 describes the two software-based turbulence mitigation methods. In Section 3 both methods are evaluated on real-world data containing varying amounts of turbulence. Finally, conclusions will be drawn in Section 4.

\section{TURBULENCE MITIGATION METHODS}

This section describes two turbulence mitigation methods developed by TNO for different turbulence conditions; low and medium turbulence.

\subsection{Low turbulence mitigation (method 1)}

An overview of the low turbulence mitigation method is depicted in Figure 1.

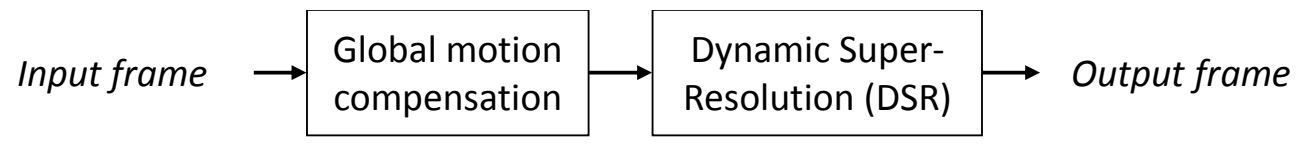

Figure 1. Flow diagram of method for low turbulence mitigation.

This method performs first "Global motion compensation" by estimating the translation between following frames and compensating for this translation ${ }^{6}$. After this step "Dynamic Super-Resolution (DSR)" is applied, which consists of backprojection of residual intensity differences on a high-resolution (HR) pixel grid ${ }^{7}$. Initialization of DSR is done with a bilinear interpolation of the first frame. For the experiments in this paper this HR grid is the same as the pixel grid of the input frame.

\subsection{Medium turbulence mitigation (method 2)}

An overview of the medium turbulence mitigation method is depicted in Figure 2.

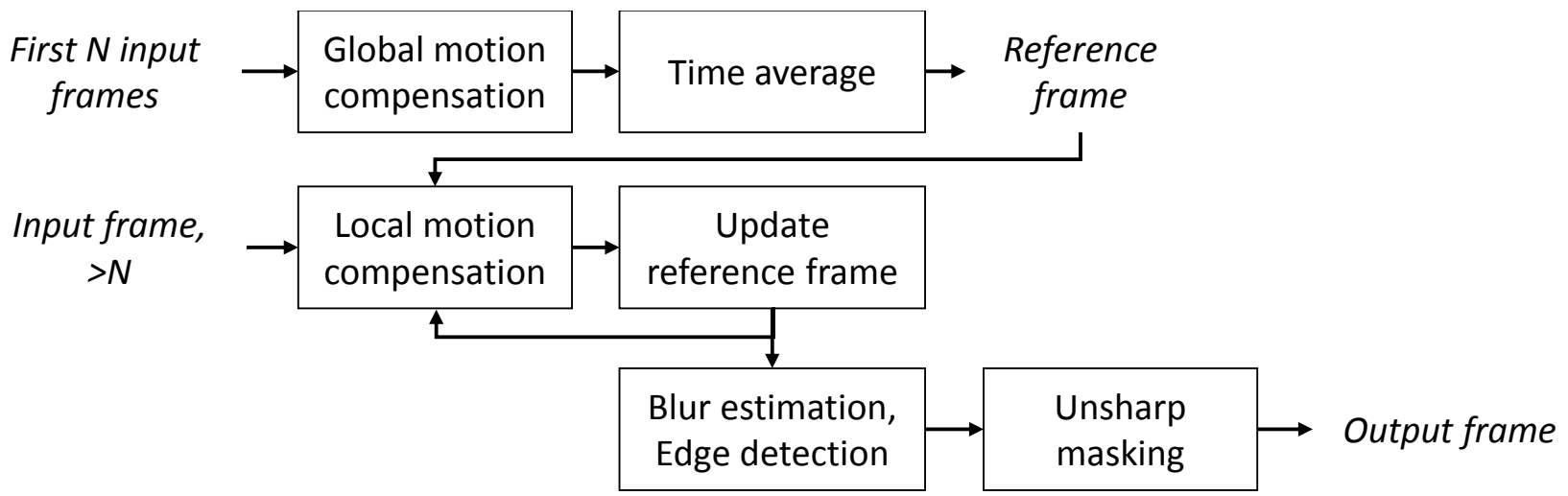

Figure 2. Flow diagram of method for medium turbulence mitigation.

This method can be split up in globally three parts: global estimation (first row), local motion compensation + update (second row), adaptive sharpening (third row). 


\section{Global estimation}

Initialization consists of a global motion (translation) compensation of the first $N$ input frames followed by a time average of these frames. This result is called the "Reference frame" and initializes the "Local motion compensation".

\section{Local motion compensation + update}

Local motion compensation is done by first calculating the optical flow ${ }^{8}$ between a new input frame and the reference frame. Optical flow estimates a per pixel local translation. When the new input frame is compensated with this local motion it is used to update the reference frame. Typically with a factor of 0.1 .

\section{Adaptive sharpening}

In the last step of the method "adaptive sharpening" is performed. First a "Blur estimation" is done using the method from Bouma et al. ${ }^{9}$ which gives a precise and un-biased result. In parallel an "Edge detection" is performed. Finally unsharp masking is done based on the estimated blur. A more pronounced unsharp masking is performed on the edges.

\section{RESULTS ON REAL-WORLD DATA}

\subsection{Real-world data under varying turbulence conditions}

For the experiments three different image sequences are used:

1. Very low turbulence conditions. This sequence is captured with a full-HD (1920x1080) daylight camera and contains part of a runway.

2. Low turbulence conditions. This sequence is captured with an infrared (IR) camera during the Fatmose trail $^{10}$ over a distance of $15.7 \mathrm{~km}$ and has a resolution of $640 \mathrm{x} 480$ pixels.

3. Medium turbulence conditions. This sequence $(1032 \times 264)$ is captured with an IR camera during a NATO SET-165 trial in Dayton, USA, beginning of October $2011^{11}$. The distance over which the imagery is captured, is approximately $7 \mathrm{~km}$.

\subsection{Results}

All results are presented in the figures below ordered from very low to medium turbulence conditions. 

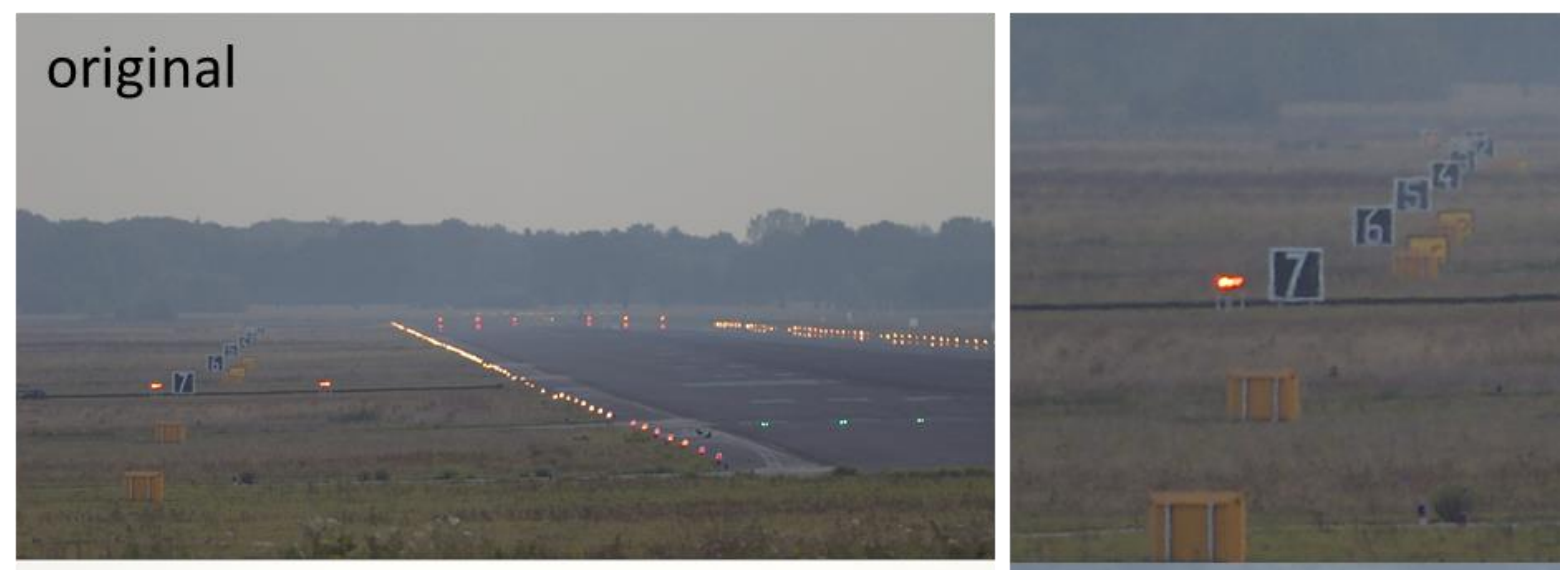

\section{low mitigation}

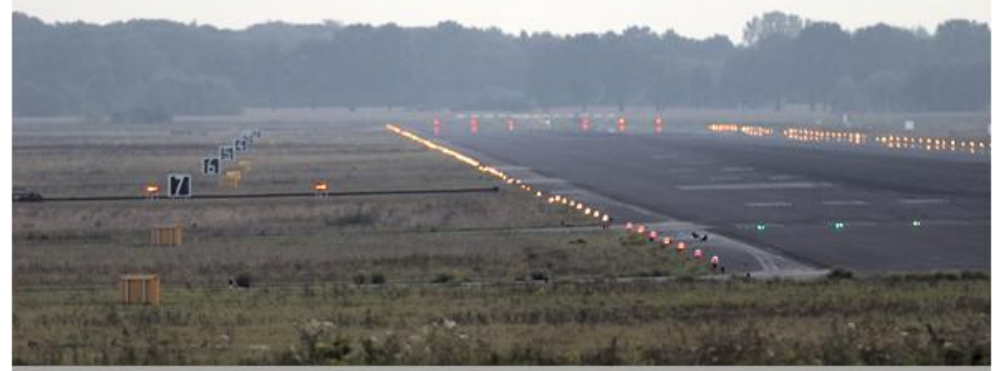

\section{medium mitigation}
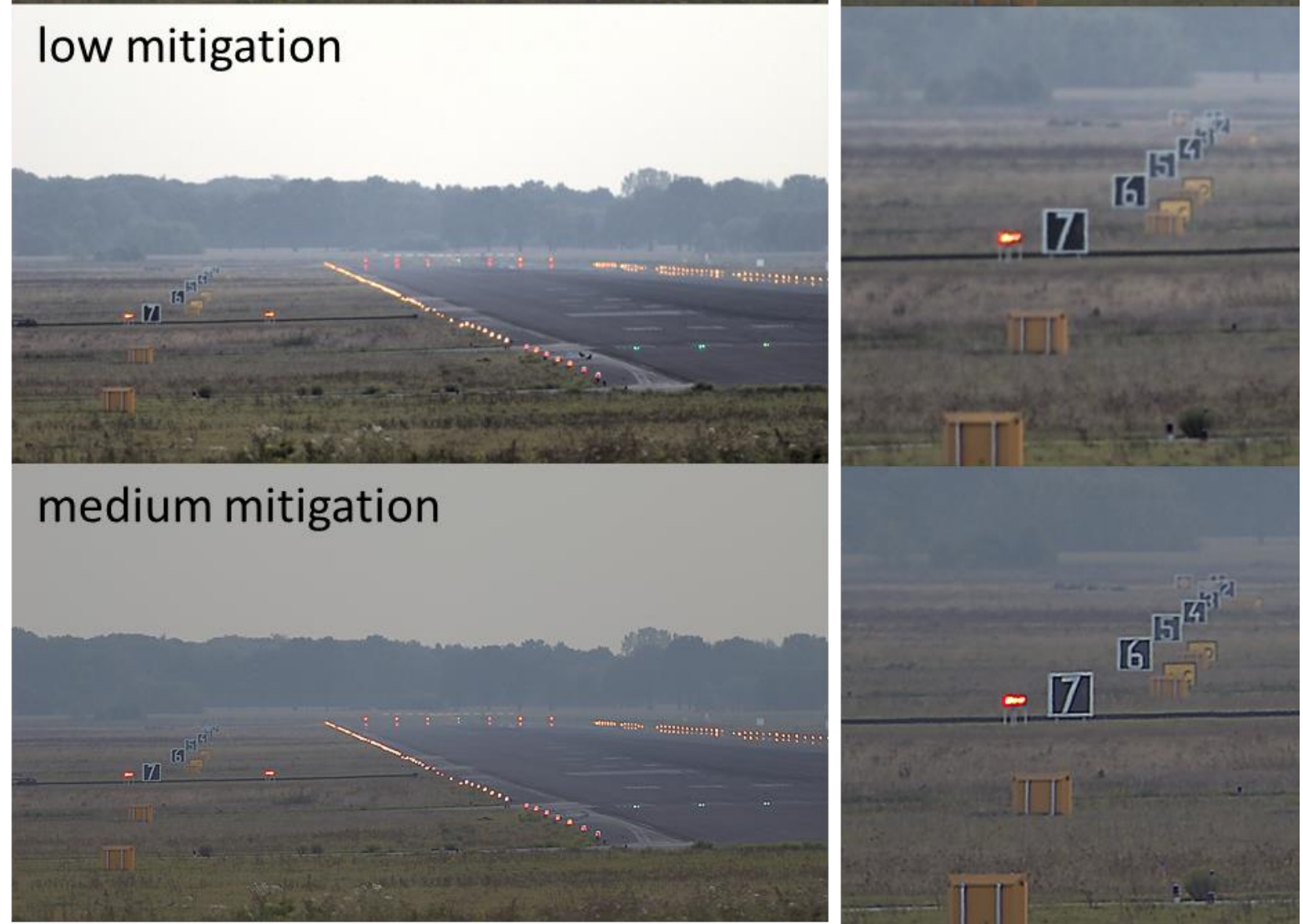

Figure 3. Turbulence mitigation results on the "very low turbulence sequence". Top row: original frame, middle row: result low turbulence mitigation (method 1), bottom row: result medium turbulence mitigation (method 2). Imagery courtesy of Adimec Advanced Image Systems.

From the results in Figure 3 it is clear that both turbulence mitigation methods produce images with more contrast and sharpness. Method 1 results in more contrast, while method 2 results in more sharpness. The methods perform equally well concerning motion compensation, because this sequence contains hardly any motion. The sequence is sharpness limited which is nicely corrected with method 2. 


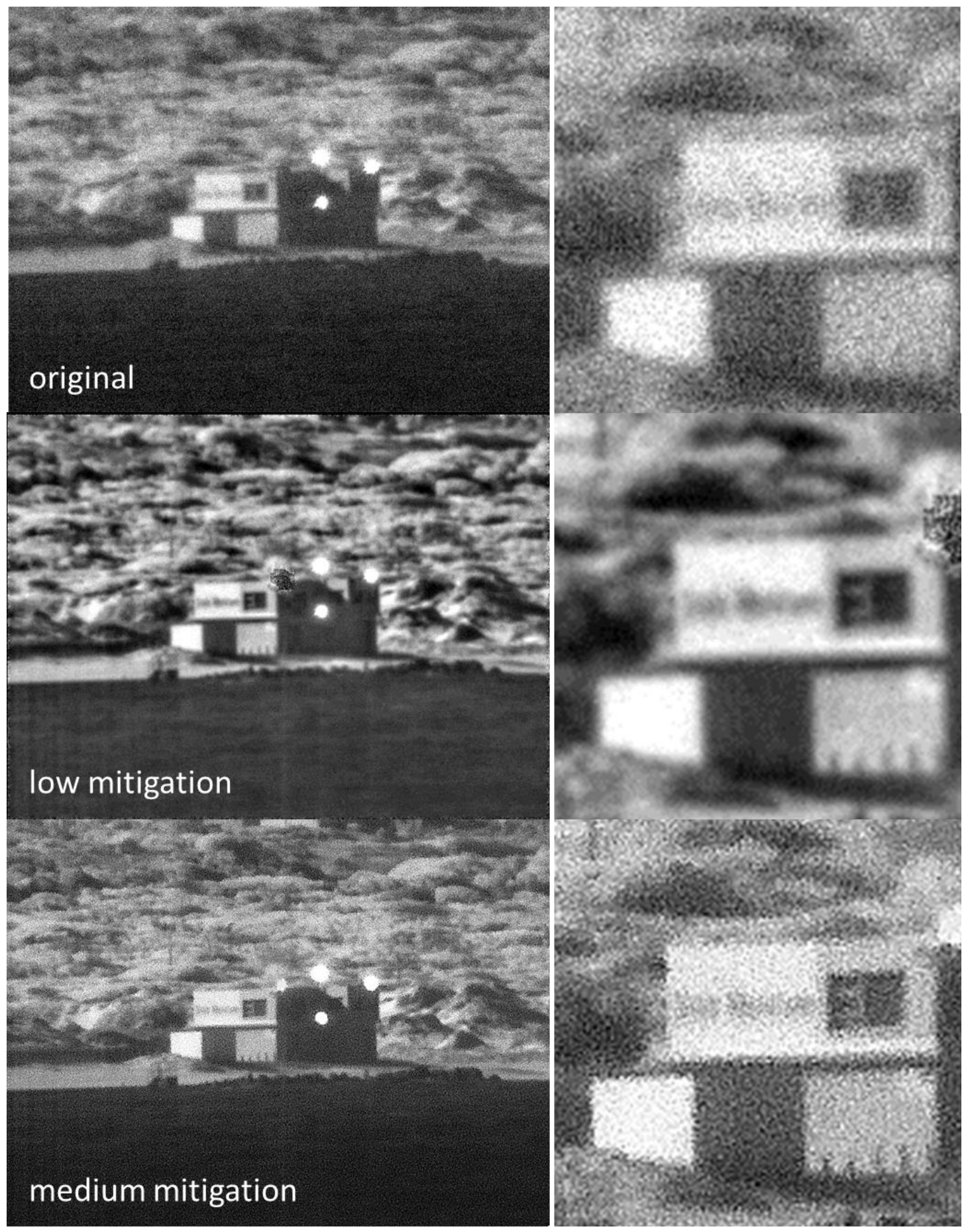

Figure 4. Turbulence mitigation results on the "low turbulence sequence". Top row: original frame, middle row: result low turbulence mitigation (method 1), bottom row: result medium turbulence mitigation (method 2 ).

From the results in Figure 4 it is clear that both turbulence mitigation methods produce very different results. Method 1 results in imagery with more contrast and less noise. Method 2 results in more sharpness and contrast (also due to ringing), but doesn't adequately reduce the noise. Tuning of the parameters (especially noise reduction versus local motion correction) should give better results, but was not performed to keep the same settings for all sequences. Both 
methods perform equally well concerning motion compensation. The sequence is noise limited which is nicely corrected by method 1 .

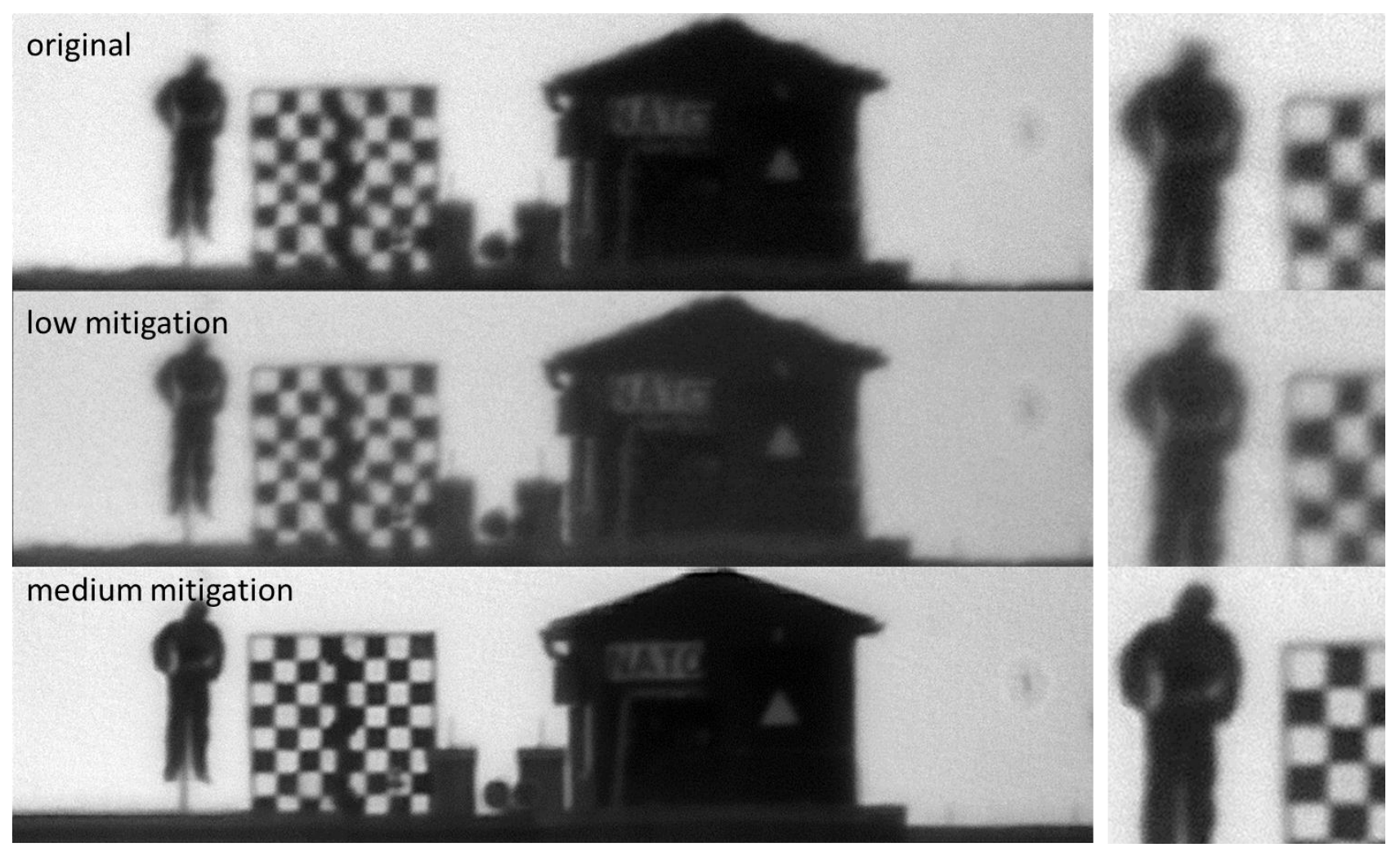

Figure 5. Turbulence mitigation results on the "medium turbulence sequence". Top row: original frame, middle row: result low turbulence mitigation (method 1), bottom row: result medium turbulence mitigation (method 2). Courtesy of NATO SET-165 group.

From the results in Figure 5 it is clear that Method 2 produces the best result concerning sharpness and motion compensation. Method 1 results in slightly more blurry imagery than the original imagery because it is not able to compensate for the local motion which is present here. Method 2 results in more sharpness and a good motion compensation, which can be observed specifically at the squares of the checkerboard. The sequence is local motion and sharpness limited which is nicely corrected by method 2 .

\section{CONCLUSIONS AND RECOMMENDATIONS}

To mitigate the effects of atmospheric turbulence different approaches can be used. In general, the complexity of these approaches increases with the amount of turbulence. The experiments in this paper are in line with this observation; method 1 performs well on low turbulence conditions, while the performance on medium turbulence conditions, with locally varying motion, is not good. Method 2 performs well on low and medium turbulence conditions, but performs less when much noise is present. 
From Section 2 it is clear that the complexity of method 2 is higher than that of method 1 . This results in that method 2 needs more processing time per frame. The decision "which turbulence mitigation method to use" will therefore not only depends on the expected turbulence conditions, but also on how it is used and how much processing power is available.

Although a lot of advances are made in the field of turbulence mitigation in recent years, there remain a lot of challenges as well. The most important challenge is to develop methods that can cope with strong turbulence conditions. This requires 1) estimating and compensating blur on a local level $\left.{ }^{9}, 2\right)$ estimating and compensating motion on a local level, 3) selecting the best temporal information on a local level. Furthermore such a method should be able to process imagery that might contain a variety of scenes, ranging from static scenes to scenes with moving objects.

\section{ACKNOWLEDGEMENTS}

The authors would like to thank the NATO SET-165 group and Adimec Advanced Image Systems for the availability of the imagery.

\section{REFERENCES}

[1] Jong, A. N. de., Schwering, P. B. W., Benoist, K. W., Gunter, W. H., Vrahimis, G.., October, F. J., "Long-term measurements of atmospheric point-spread functions over littoral waters as determined by atmospheric turbulence,” Proc. SPIE Infrared Imaging Systems: Design, Analysis, Modeling, and Testing XXIII 8355 (2012).

[2] Schwering, P. B.., Kunz, G. J., "Infrared scintillation effects over sea," SPIE’s 1995 Symposium on OE/Aerospace Sensing and Dual Use Photonics 2471, 204-215 (1995).

[3] Roggeman, M. C.., Welsh, B., "Imaging through turbulence," CRC Press, Boca Raton, USA (1996).

[4] Eekeren, A. W. M. van., Schutte, K., Dijk, J., Schwering, P. B. W., Iersel, M. van., Doelman, N. J., "Turbulence Compensation: an overview," Proc. SPIE Infrared Imaging Systems XXIII 8355 (2012).

[5] Eekeren, A. W. M. van., Huebner, C. S., Dijk, J., Schutte, K.., Schwering, P. B. W., "Evaluation of turbulence mitigation methods," Proc. SPIE Infrared Imaging Systems XXV 9071 (2014).

[6] Pham, T. Q., Bezuijen, M., Vliet, L. J. van., Schutte, K.., Hendriks, C. L. L., "Performance of optimal registration estimators," Proc. SPIE Visual Information Processing XIV 5817, 133-144 (2005).

[7] Schutte, K., Lange, D. J. J. de., Broek, S. P. van den., "Signal conditioning algorithms for enhanced tactical sensor imagery," Proc. SPIE Infrared Imaging Systems XIV 5076 (2003).

[8] Horn, B. K. P.., Schunck, B. G., "Determining optical flow," Artificial Intelligence 17, 185-203 (1981).

[9] Bouma, H., Dijk, J.., Eekeren, A. W. M. van., "Precise local blur estimation based on the first-order derivative," Proc. SPIE Visual Information Processing XXI 8399 (2012).

[10] Jong, A. N. de., Fritz, P. J., Benoist, K. W., Eijk, A. M. J. van., Schwering, P. B. W., "Preliminary results of the FATMOSE atmospheric propagation trials in the False Bay, South Africa, November 2009 - July 2010," Proc. SPIE Optics in Atmospheric Propagation and Adaptive Systems XIII 7828 (2010).

[11] Velluet, M. T., Vorontsov, M., Espinola, R., Marchi, G., Nicolas, S., Schwering, P.., Riker, J., "Turbulence characterization and image processing data sets from a NATO RTO SET 165 trial in Dayton, OH, USA," Proc. SPIE Atmospheric Propagation IX 8380 (2012). 